\title{
THE WELL-POSEDNESS OF A HYPERBOLIC STEFAN PROBLEM*
}

\author{
BY \\ DENING LI ${ }^{1}$ \\ University of Colorado at Boulder
}

1. Introduction. Recently, several authors have considered the phase change problem governed by the hyperbolic heat transfer model $[2,5,6,7]$. Let $q$ be the heat flux, $T$ the temperature, $\tau$ the relaxation time, $k$ the thermal conductivity, $\rho$ the density, $c$ the specific heat, $\alpha=k /(\rho c)$ the diffusivity. The hyperbolic heat transfer model is obtained by replacing the classical Fourier heat-conduction law

$$
q=-k T_{x}
$$

with the first-order relaxation relation

$$
\tau q_{t}+q=-k T_{x}
$$

Combining (1.2) and the energy conservation law

$$
c \rho T_{t}=-q_{x},
$$

one gets the governing equations of the hyperbolic heat transfer model

$$
\left\{\begin{array}{l}
\tau q_{t}+q+k T_{x}=0 \\
c \rho T_{t}+q_{x}=0
\end{array}\right.
$$

or equivalently, the telegrapher's equation

$$
\tau T_{t t}+T_{t}=\alpha T_{x x}
$$

If $\tau=0$, the telegrapher's equation (1.5) is reduced to the usual heat equation

$$
T_{t}-\alpha T_{x x}=0 .
$$

As to the relation between the solutions $T_{\tau}$ and $T_{0}$ of (1.5) and (1.6) with compatible initial data, it has long been known that $T_{\tau} \rightarrow T_{0}$ uniformly when $\tau \rightarrow 0$, see, e.g. [3].

For the classical heat transfer model (1.6), the one-phase Stefan problem consists of finding the temperature $T(x, t)$ and a function $\varphi(t)$ such that (1.6) is satisfied in the domain $x<\varphi(t)$ and on the free boundary $x=\varphi(t)$, the following conditions are satisfied:

$$
\begin{gathered}
T(\varphi(t), t)=0, \\
\rho H \varphi^{\prime}(t)=q .
\end{gathered}
$$

*Received January 29, 1988.

'Current address: Mathematics Department, West Virginia University, Morgantown, WV 26506.

(C)1989 Brown University 
The two-phase Stefan problem is formulated similarly.

The Stefan problem and the related problems for the heat equation (1.6) or more general equations have been the objects of extensive study. Therefore, it is only natural that the problems of Stefan type for the hyperbolic heat transfer model (1.4) are recently becoming of interest to many mathematicians.

In [6] was given a formulation of the hyperbolic Stefan problem based upon the traditional assumption that the temperatures on the two sides of the phase change boundary are prescribed and equal. Also, an explicit solution was given in [6] where the phase change front propagates faster than sound speed and consequently is physically unacceptable.

Partly in order to avoid this difficulty, Greenberg in [2] suggested another formulation of the phase change condition based upon the Rankine-Hugoniot conditions for the conservation laws.

In [5] was given a weak formulation of the two-phase Stefan problem, including mushy domains. The problem posed and resolved there is different from the one considered here. The relaxation times in the two phases are adjusted to make it possible to study the problem in the framework of abstract operators.

In this paper, we study the Stefan problem for the hyperbolic heat model in the classical formulation of [6]. For both the one-phase and the two-phase problem, we get the local and global solution. The conditions to guarantee the global existence in this paper are only sufficient ones, in comparison with the local existence conditions which are necessary. So the conditions for global solutions should be able to be relaxed. It remains open as to what extent these conditions could be relaxed.

The outline of this paper is as follows.

In $\S 2$, we prove the local existence and uniqueness results for the initial boundary Stefan problem, and we give the mathematical explanation of the example given in [6].

In $\S 3$, using similar techniques as in [2], we prove the global existence of solution for the Stefan problem under certain assumptions upon the data.

In $\S 4$, we give a brief discussion of the two-phase Stefan problem.

2. One-phase problem, Local solution. The one-phase Stefan problem for the hyperbolic heat transfer model consists in solving the following free boundary problem:

$$
\begin{gathered}
\left\{\begin{array}{l}
\tau q_{t}+q+k T_{x}=0, \\
c \rho T_{t}+q_{x}=0,
\end{array} \quad x_{0}<x<\varphi(t), \quad t>0,\right. \\
\left\{\begin{array}{l}
T(x, t)=0, \\
\rho H \varphi^{\prime}(t)=q(x, t),
\end{array} \quad x=\varphi(t), \quad t>0,\right.
\end{gathered}
$$

where $H$ is the latent heat, and $\varphi(0)=0$.

We may impose boundary conditions on the fixed boundary $x=x_{0} \leq 0$. They could take the following forms, depending on whether temperature or heat flux is given [6]:

- Imposed temperature boundary condition:

$$
T\left(x_{0}, t\right)=T_{\#}(t), \quad t>0 ;
$$


- Imposed flux boundary condition:

$$
q\left(x_{0}, t\right)=q_{\#}(t), \quad t>0
$$

- Convective boundary condition:

$$
q\left(x_{0}, t\right)=h\left[q_{\#}(t)-T\left(x_{0}, t\right)\right], \quad t>0 .
$$

If $x_{0}=0$, then no initial condition is needed. If $x_{0}<0$, we have to give the initial values:

$$
T(x, 0)=T_{0}(x), \quad q(x, 0)=q_{0}(x), \quad x_{0} \leq x \leq 0 .
$$

In the following, we'll discuss separately the cases of $x_{0}<0$ and $x_{0}=0$.

2.1. Case 1: $x_{0}<0$. Since we consider only local solutions in this section, the well-posedness of the problem (2.1), (2.2), (2.3), (2.6) (or (2.3) substituted by (2.4) or (2.5)) is determined completely from the well-posedness of the fixed boundary problem (2.1), (2.3), (2.6) and the free boundary problem (2.1), (2.2), (2.6) separately, because of the finite propagation speed. It is easily checked that the fixed boundary problem (2.1), (2.3), (2.6) is always well-posed. (It is also true with (2.3) substituted by (2.4) or (2.5).) Therefore we need only consider the following initialfree-boundary problem:

$$
\begin{gathered}
\left\{\begin{array}{l}
\tau q_{t}+k T_{x}+q=0, \\
c \rho T_{t}+q_{x}=0,
\end{array} \quad x<\varphi(t), \quad t>0,\right. \\
\left\{\begin{array}{l}
T(x, t)=0, \\
\rho H \varphi^{\prime}(t)=q(x, t),
\end{array} \quad x=\varphi(t), \quad t>0,\right. \\
T(x, 0)=T_{0}(x), \quad q(x, 0)=q_{0}(x) .
\end{gathered}
$$

We have the following theorem.

Theorem 2.1. Let $T_{0}, q_{0} \in C^{1}(-\infty, 0]$. Suppose at $x=0, t=0$, the compatible condition

$$
c \rho \partial_{x} T_{0}(0) q_{0}(0)=\rho H \partial_{x} q_{0}(0)
$$

is satisfied and also

$$
\left|q_{0}(0)\right|<\rho H(\alpha / \tau)^{1 / 2}, \alpha=\frac{k}{c \rho} .
$$

Then $\exists t_{0}>0$ such that in $\left[0, t_{0}\right],(2.7),(2.8),(2.9)$ has a unique solution $(T, q, \varphi) \in$ $C^{1} \times C^{1} \times C^{2}$.

Remark. The condition (2.11) implies that at $t=0$, the speed of phase change front should be less than the characteristic speed. This condition is necessary in the sense that there is no solution depending only on $x-(\alpha / \tau)^{1 / 2} t$, as pointed out in [6]. And if $\left|\varphi^{\prime}(0)\right|>(\alpha / \tau)^{1 / 2}$, then $x=\varphi(t)$ would be space-like for small $t$. Therefore, besides the condition on $\varphi(t)$ in (2.8), one should impose two conditions (for $\varphi^{\prime}(0)>(\alpha / \tau)^{1 / 2}$ ) or no condition (for $\varphi^{\prime}(0)<-(\alpha / \tau)^{1 / 2}$ ) on $x=\varphi(t)$ for $(q, T)$ to get a well-posed problem. Otherwise, one would lose either uniqueness or existence of the solution. This explains the physically unacceptable explicit solution example in [6]. Actually, in the example of [6] where (2.11) is violated, the solution is not unique. 
Proof of Theorem 2.1.

i) First, as in [2], we reduce (2.7)-(2.9) into dimensionless form by making the following substitutions:

$$
\begin{cases}\partial_{t}=\frac{1}{\tau} \partial_{\bar{t}}, & \partial_{x}=\left(\frac{c \rho}{k \tau}\right)^{1 / 2} \partial_{\bar{x}}, \\ T=\frac{H}{c} \bar{T}, & q=H\left(\frac{k \rho}{c \tau}\right)^{1 / 2} \bar{q}, \quad \varphi=\left(\frac{k \tau}{c \rho}\right)^{1 / 2} \bar{\varphi} .\end{cases}
$$

Omitting the bar over the new variables and letting

$$
A=T+q, \quad B=T-q,
$$

then (2.7)-(2.9) become

$$
\begin{gathered}
\left\{\begin{array}{l}
A_{t}+A_{x}+\frac{1}{2}(A-B)=0, \\
B_{t}-B_{x}+\frac{1}{2}(B-A)=0,
\end{array}\right. \\
\left\{\begin{array}{l}
(A+B)(x, t)=0, \\
\varphi^{\prime}(t)=\frac{1}{2}(A-B)(x, t),
\end{array} \text { in } x<\varphi(t),\right. \\
A(x, 0)=A_{0}(x), \quad B(x, 0)=B_{0}(x), \quad \varphi(0)=0 .
\end{gathered}
$$

ii) Secondly, we perform the following transformation:

$$
\bar{x}=x-\varphi(t), \quad \bar{t}=t
$$

to fix the free boundary in $(2.14)-(2.16)$. Since

$$
\left\{\begin{array}{l}
\partial_{t}=\partial_{\bar{t}}-\varphi^{\prime}(\bar{t}) \partial_{\bar{x}} \\
\partial_{x}=\partial_{\bar{x}}
\end{array}\right.
$$

omitting the bar in the new notation, we get the following equivalent fixed boundary problem for the unknown $(T, q, \varphi)$ :

$$
\begin{gathered}
\left\{\begin{array}{l}
A_{t}+\left(1-\varphi^{\prime}(t)\right) A_{x}+\frac{1}{2}(A-B)=0, \\
B_{t}-\left(1+\varphi^{\prime}(t)\right) B_{x}+\frac{1}{2}(B-A)=0,
\end{array}\right. \\
\qquad\left\{\begin{array}{l}
A(0, t)+B(0, t)=0, \\
\varphi^{\prime}(t)=\frac{1}{2}(A-B)(0, t),
\end{array}\right. \\
A(x, 0)=A_{0}(x), \quad B(x, 0)=B_{0}(x), \quad \varphi(0)=0 .
\end{gathered}
$$

iii) Now, from assumption (2.11), we know that for $\left|\varphi^{\prime}(t)-\varphi^{\prime}(0)\right|$ sufficiently small, $x=0$ is noncharacteristic for (2.8). And the linearized problem of $(2.18)-(2.20)$ is well-posed. Therefore, the nonlinear problem $(2.18)-(2.20)$ can be solved by the usual technique of integration along characteristics and linear iteration. Hence the proof of Theorem 2.1 is complete. For details, one may refer to, e.g., [1] or [4].

Corollary 2.1. If $T_{0}(x), q_{0}(x) \in C^{\infty}$, and $C^{\infty}$ compatible conditions are satisfied, then the solution in Theorem 2.1 is actually in $C^{\infty}\left[0, t_{0}\right]$.

2.2. Case 2: $x_{0}=0$. In this case, we don't impose the initial condition (2.6) and consider the free boundary problem $(2.1),(2.2)$ combined with one of the boundary conditions in (2.3), (2.4), and (2.5). We have the following. 
Theorem 2.2. i) Assume $q_{\#}(t), T_{\#}(t) \in C^{1}[0, \infty)$. At $t=0, x=0, q(x, t)$ determined from (2.4) or (2.5) satisfies

$$
0<q(0,0)<\rho H(\alpha / \tau)^{1 / 2}
$$

Then $\exists t_{0}>0$ such that in $\left[0, t_{0}\right]$, the problem $(2.1),(2.2),(2.4)$ or the problem (2.1), (2.2), (2.5) has a unique solution $(T, q, \varphi) \in C^{1} \times C^{1} \times C^{2}$;

ii) The problem (2.1), (2.2), (2.3) is not well-posed.

Proof of Theorem 2.2.

i) We will consider only the Problem (2.1), (2.2), (2.4). The problem (2.1), (2.2), (2.5) can be treated exactly the same way.

As in the proof of Theorem 2.1, we first transform (2.1), (2.2), (2.4) into the dimensionless and diagonal form, which can be written as

$$
\begin{gathered}
\left\{\begin{array}{l}
A_{t}+A_{x}+\frac{1}{2}(A-B)=0, \\
B_{t}-B_{x}+\frac{1}{2}(B-A)=0,
\end{array}\right. \\
\left\{\begin{array}{l}
(A+B)(x, t)=0, \\
\varphi^{\prime}(t)=\frac{1}{2}(A-B)(x, t)=0,
\end{array}\right. \\
(A-B)(0, t)=2 q_{*}(t),
\end{gathered}
$$

where $\varphi(0)=0$ and the condition (2.21) becomes

$$
0<q_{*}(0)<1 \text {. }
$$

Now we perform the transformation $(t, x) \mapsto(t, y)$ :

$$
y=\frac{x t}{\varphi(t)}
$$

From (2.23), (2.24), (2.25) we know $\varphi^{\prime}(0)>0$, hence (2.26) is not singular for small $t$ when $\varphi(t)$ is continuous. In $(t, y)$ coordinates $(2.22),(2.23),(2.24)$ become

$$
\begin{gathered}
\left\{\begin{array}{l}
A_{t}+a(\varphi, y, t) A_{y}+\frac{1}{2}(A-B)=0, \\
B_{t}+b(\varphi, y, t) B_{y}+\frac{1}{2}(B-A)=0,
\end{array}\right. \\
(A+B)(y, t)=0, \quad \text { on } y=t, \\
\varphi^{\prime}(t)=\frac{1}{2}(A-B)(y, t)=A(y, t), \quad \text { on } y=t, \\
(A-B)(0, t)=2 q_{*}(t),
\end{gathered}
$$

where

$$
\begin{aligned}
& a(\varphi, y, t)=\frac{y\left(\varphi-\varphi^{\prime}(t) t\right)}{t \varphi}+\frac{t}{\varphi}, \\
& b(\varphi, y, t)=\frac{y\left(\varphi-\varphi^{\prime}(t) t\right)}{t \varphi}-\frac{t}{\varphi} .
\end{aligned}
$$

Now $(2.27)-(2.30)$ is a nonlinear boundary problem in the known angular domain. From assumption (2.25), we have

$$
0<\varphi^{\prime}(0)<1 .
$$


Therefore, near $t=0, a(\varphi, y, t)$ and $b(\varphi, y, t)$ are nonsingular and

$$
\left.a\right|_{t=0}=\frac{1}{\varphi^{\prime}(0)},\left.\quad b\right|_{t=0}=-\frac{1}{\varphi^{\prime}(0)} .
$$

We solve (2.27)-(2.30) locally by linear iteration and integration along characteristics.

By (2.31), (2.32), we see that the linearization of (2.27), (2.28), (2.30) at $t=0$

$$
\begin{aligned}
& \left\{\begin{array}{l}
A_{t}+\left(\varphi^{\prime}(0)\right)^{-1} A_{y}+\frac{1}{2}(A-B)=0, \\
B_{t}-\left(\varphi^{\prime}(0)\right)^{-1} B_{y}+\frac{1}{2}(B-A)=0,
\end{array} \quad 0<y<t,\right. \\
& (A+B)(t, t)=0, \\
& (A-B)(0, t)=2 q_{*}(t),
\end{aligned}
$$

is a typical boundary value problem in angular domain discussed in [4], with two boundaries $y=0, y=t$ noncharacteristic. For the problem (2.33)-(2.35), following [4], we can compute the characterizing matrix

$$
H=\left(\begin{array}{cc}
0 & -1 \\
1 & 0
\end{array}\right)
$$

and

$$
H_{1}=\left(\begin{array}{cc}
0 & -\left(1-\varphi^{\prime}(0)\right) \\
\left(1+\varphi^{\prime}(0)\right)^{-1} & 0
\end{array}\right) .
$$

Hence, we have $\operatorname{det}(I-H) \neq 0$ and $\left|H_{1}\right|_{\min }=\min \left\{\left(1+\varphi^{\prime}(0)\right)^{-1}, 1-\varphi^{\prime}(0)\right\}<1$ by (2.31). From an extension of the results of $\mathrm{Li}$ and $\mathrm{Yu}$ by $\mathrm{Zhao}$ in [8], we know the linearized problem (2.33)-(2.35) has a unique $C^{1}$ solution, using the method of integration along the characteristics.

With the solution $\left(A_{1}, B_{1}\right)$ of the linearized problem (2.33)-(2.35), we can solve $\varphi_{1}(t) \in C^{2}$ from (2.29), and iteratively we get the sequence $\left(A_{j}, B_{j}, \varphi_{j}\right)$ and we can prove its convergence for $t \leq T_{0}$ small. This completes the proof of the first part of Theorem 2.2.

For the second part of Theorem 2.2, we notice that, from (2.1)-(2.3), at point $x=$ $\varphi(t)=t=0$, we couldn't determine the value of $q$. Actually, we can choose $q(0,0)$ arbitrarily, i.e., $\varphi^{\prime}(0)$ can be chosen arbitrarily. If we choose $q(0,0)>\rho H(\alpha / \tau)^{1 / 2}$, then we can impose an extra boundary condition on $x=\varphi(t)$, e.g., $q(\varphi(t), t)=$ $q(0,0)$, and the resultant free boundary problem can be proven to be well-posed just as above. Therefore, the solution of $(2.1)-(2.3)$ is not unique. So the problem is not well-posed.

Remark. For the second part of Theorem 2.2, even if we choose $0<q(0,0)<$ $\rho H(\alpha / \tau)^{1 / 2}$, the resulting linearized problem is not well-posed. To see this, we can compute the corresponding characterizing matrix $H$ as in the proof of the first part of the Theorem, and find

$$
H=\left(\begin{array}{ll}
0 & 1 \\
1 & 0
\end{array}\right) .
$$

So $\operatorname{det}(I-H)=0$. Consequently, we see that the problem is not well-posed.

3. One-phase problem, Global solution. In this section, we will discuss the global existence of solutions for the problems considered in $\S 2$. 
We will restrict ourselves to discussing only the problem where on the fixed boundary $x=x_{0}$, the imposed temperature condition is given.

As in $\S 2$, in dimensionless form, the problem for the Riemann invariants $A, B$ can be written as

$$
\begin{gathered}
\left\{\begin{array}{l}
A_{t}+A_{x}+\frac{1}{2}(A-B)=0, \\
B_{t}-B_{x}+\frac{1}{2}(B-A)=0,
\end{array}\right. \\
\left\{\begin{array}{l}
(A+B)(x, t)=0, \\
\varphi^{\prime}(t)=A(x, t),
\end{array} \quad \text { in } x_{0}<x<\varphi(t),\right. \\
\quad(A+B)\left(x_{0}, t\right)=2 T_{\#}(t), \\
A(x, 0)=A_{0}(x), \quad B(x, 0)=B_{0}(x), \quad \varphi(0)=0 .
\end{gathered}
$$

According to Theorem 2.2, we should have $x_{0}<0$ in (3.1)-(3.4). We have

Theorem 3.1. Assume: (i) $A_{0}, B_{0} \in C^{\infty}\left[x_{0}, 0\right], T_{\#} \in C^{\infty}[0, \infty)$; (ii) $T_{\#}^{\prime}(t) \geq 0$, $A_{0}^{\prime}<0, B_{0}^{\prime}<0,0<A(0,0)<1$; (iii) $C^{\infty}$ compatible conditions are satisfied at $(0,0)$ and $\left(x_{0}, 0\right)$.

Then (3.1)-(3.4) has a unique solution $(A, B, \varphi) \in C^{\infty} \times C^{\infty} \times C^{\infty}$ for all $t>0$, and the solution satisfies

$$
A(\varphi(t), t)>0, \quad A_{x}<0, \quad B_{x}<0, \quad 0<\varphi^{\prime}(t)<1 .
$$

Proof of Theorem 3.1. From Theorem 2.1, we know that under the assumption of Theorem 3.1, a unique solution exists and if the solution exists in some interval $\left[0, t_{0}\right]$ where the condition $0<\varphi^{\prime}\left(t_{0}\right)<1$ is again satisfied, then we can solve the corresponding free boundary problem beginning from $t=t_{0}$. Therefore, in order to prove Theorem 3.1 , one has only to show that in any interval $\left[0, t_{0}\right]$ where a $C^{\infty}$ solution exists, we can find a $\delta$ such that

$$
\left|\varphi^{\prime}\left(t_{0}\right)\right|<1-\delta .
$$

Equation (3.6) implies that the corresponding linearized problem has uniformly noncharacteristic boundaries. Hence the global solution can be obtained with linear iteration step by step.

Actually, under the assumption of (ii) in Theorem 3.1, we can not only prove (3.6), but also

$$
\varphi^{\prime}(t)>0, \quad A_{x}(x, t)<0, \quad B_{x}(x, t)<0 .
$$

To prove (3.7), we use the technique employed in [2]. Let $t^{*}$ be the smallest $t \in\left[0, t_{0}\right]$ such that (3.7) is violated. Differentiating $A(x, t)$ on $x=\varphi(t)$ and employing (3.1), (3.2), one gets

$$
\begin{aligned}
\frac{d A(\varphi(t), t)}{d t} & =A_{t}(\varphi(t), t)+A_{x}(\varphi(t), t) \varphi^{\prime}(t) \\
& =-A_{x}(\varphi(t), t)-A(\varphi(t), t)-A_{x}(\varphi(t), t) A(\varphi(t), t) \\
& =-A_{x}(\varphi(t), t)(1+A(\varphi(t), t))-A(\varphi(t), t) .
\end{aligned}
$$

Hence we have

$$
\frac{d A(\varphi(t), t)}{d t} \geq-A(\varphi(t), t), \text { in }\left[0, t^{*}\right]
$$


Consequently

$$
A(\varphi(t), t) \geq A(0,0) e^{-t}>0 .
$$

Let $\mathscr{A}=A_{x}, \mathscr{B}=B_{x}$. Then $\mathscr{A}, \mathscr{B}$ satisfy the following:

$$
\begin{aligned}
& \left\{\begin{array}{l}
\mathscr{A}_{t}+\mathscr{A}_{x}+\frac{1}{2}(\mathscr{A}-\mathscr{B})=0, \\
\mathscr{B}_{t}-\mathscr{B}_{x}+\frac{1}{2}(\mathscr{B}-\mathscr{A})=0,
\end{array} \quad \text { in } x_{0}<x<\varphi(t),\right. \\
& \mathscr{B}-\mathscr{A}=2 T_{\#}^{\prime}(t), \quad \text { on } x=x_{0} \text {, } \\
& \mathscr{B}=\frac{1-A}{1+A} \mathscr{A}, \quad \text { on } x=\varphi(t), \\
& \mathscr{B}(x, 0)=B_{0}^{\prime}(x), \quad \mathscr{A}(x, 0)=A_{0}^{\prime}(x) .
\end{aligned}
$$

As in [2], we rewrite (3.8) into

$$
\left\{\begin{array}{l}
\left(\partial_{t}+\partial_{x}\right)\left(e^{t / 2} \mathscr{A}\right)=\frac{1}{2} e^{t / 2} \mathscr{B} \\
\left(\partial_{t}-\partial_{x}\right)\left(e^{t / 2} \mathscr{B}\right)=\frac{1}{2} e^{t / 2} \mathscr{A} .
\end{array}\right.
$$

Since in $\left[0, t^{*}\right), \mathscr{A}<0, \mathscr{B}<0, A(\varphi(t), t)>0$, hence from $(3.12), \mathscr{A}\left(x, t^{*}\right)=0$ is possible only at $x=x_{0}, \mathscr{B}\left(x, t^{*}\right)=0$ is possible only at $x=\varphi\left(t^{*}\right)$. But at $x=x_{0}$, $\mathscr{A}=\mathscr{B}-2 T_{\#}^{\prime}(t)<0$ and at $x=\varphi\left(t^{*}\right), \mathscr{B}=(1-A)(1+A)^{-1} \mathscr{A}<0$. Consequently, we must have $t^{*}=t_{0}$.

Now, the linear problem (3.8)-(3.11) for $A, B$, has uniformly bounded coefficients, since $A>0$ on $x=\varphi(t)$. So the solution $A, B$ must be bounded on $\left[0, t_{0}\right]$. In particular, $T_{x}$ is bounded on $\left[0, t_{0}\right]$,

$$
\max _{\left[0, t_{0}\right]}\left|T_{x}\right| \leq C\left(t_{0}\right),
$$

where $C\left(t_{0}\right)<\infty$ for any fixed $t_{0}>0$.

On $x=\varphi(t)$, by [6], we have

$$
\varphi^{\prime \prime}(t)+\varphi^{\prime}(t)=T_{x}(\varphi(t), t)\left(\left(\varphi^{\prime}\right)^{2}-1\right) .
$$

Since $\left|T_{x}\right| \leq C\left(t_{0}\right)$, so when $\varphi^{\prime}(t) \rightarrow 1$, one should have $\varphi^{\prime \prime}(t)<0$. Therefore, there must be a $\delta>0$, such that $\varphi^{\prime}(t)<1-\delta$, where $\delta=\delta\left(t_{0}\right)>0$, for any fixed $t_{0}$. Thus Theorem 3.1 is proved.

4. Two-phase problem. In this section, we want to extend our results for the onephase problems in the preceding sections to two-phase problems. With similar notations as before, the two-phase problem is formulated as follows:

$$
\begin{aligned}
& \left\{\begin{array}{l}
\tau_{1} \partial_{t} q_{1}+k_{1} \partial_{x} T_{1}+q_{1}=0, \\
c_{1} \rho \partial_{t} T_{1}+\partial_{x} q_{1}=0,
\end{array} \quad \text { in } x<\varphi(t),\right. \\
& \left\{\begin{array}{l}
\tau_{2} \partial_{t} q_{2}+k_{2} \partial_{x} T_{2}+q_{2}=0, \\
c_{2} \rho \partial_{t} T_{2} t+\partial_{x} q_{2}=0,
\end{array} \quad \text { in } x>\varphi(t),\right. \\
& \left\{\begin{array}{l}
T_{1}(x, t)=T_{2}(x, t)=0, \\
\rho H \varphi^{\prime}(t)=\left(q_{1}-q_{2}\right)(x, t),
\end{array} \quad \text { on } \varphi(t),\right. \\
& \left\{\begin{array}{lll}
T_{1}(x, 0)=T_{10}(x), & q_{1}(x, 0)=q_{10}(x), & x<0, \\
T_{2}(x, 0)=T_{20}(x), & q_{2}(x, 0)=q_{20}(x), & x>0, \\
\varphi(0)=0 . &
\end{array}\right.
\end{aligned}
$$


Here we assume the densities $\rho$ in the two phases are equal.

For the two-phase problem (4.1)-(4.4), we have the following local result.

THEOREM 4.1. Let $T_{10}, q_{10}, T_{20}, q_{20} \in C^{1}$, and the compatible conditions are satisfied at $(0,0)$. Also is satisfied the following:

$$
\left|\left(q_{20}-q_{10}\right)(0)\right|<\min \left\{\rho H\left(\alpha_{1} / \tau_{1}\right)^{1 / 2}, \rho H\left(\alpha_{2} / \tau_{2}\right)^{1 / 2}\right\} .
$$

Then, there exists $t_{0}>0$ such that there is a unique solution $\left(T_{1}, q_{1}, T_{2}, q_{2}\right) \in C^{1}$, $\varphi \in C^{2}$ of $(4.1)-(4.4)$ in $\left[0, t_{0}\right]$.

The proof of Theorem 4.1 can be performed exactly the same way as the proof of Theorem 2.1, i.e., perform the coordinate transformation $y=x-\varphi(t)$ to fix the free boundary $x=\varphi(t)$ and then, solve the nonlinear problem by linear iteration. We omit the details here.

For the global solution of the two-phase problem, we assume

$$
\tau_{1}=\tau_{2}, \quad \alpha_{1}=\alpha_{2} .
$$

On two fixed boundaries $x=-x_{0}<0, x=x_{0}>0$, the imposed temperature conditions are given:

$$
T_{1}\left(-x_{0}, t\right)=T_{1 \#}(t), \quad T_{2}\left(x_{0}, t\right)=T_{2 \#}(t) .
$$

We introduce the Riemann invariants $A, B$ as in $\S 2$ :

$$
\left\{\begin{array}{l}
q_{j}=\sqrt{\frac{k_{j}}{\tau}}\left(A_{j}-B_{j}\right), \\
T_{j}=\sqrt{\frac{1}{c_{j} \rho}}\left(A_{j}+B_{j}\right) .
\end{array} \quad j=1,2 .\right.
$$

Then $A_{j}, B_{j}(j=1,2)$ satisfy the following:

$$
\begin{gathered}
\left\{\begin{array}{l}
\partial_{t} A_{1}+\sqrt{\frac{\alpha}{\tau}} \partial_{x} A_{1}+\frac{1}{2 \tau}\left(A_{1}-B_{1}\right)=0, \\
\partial_{t} B_{1}-\sqrt{\frac{\alpha}{\tau}} \partial_{x} B_{1}+\frac{1}{2 \tau}\left(B_{1}-A_{1}\right)=0,
\end{array}\right. \\
\left\{\begin{array}{l}
\partial_{t} A_{2}+\sqrt{\frac{\alpha}{\tau}} \partial_{x} A_{2}+\frac{1}{2 \tau}\left(A_{2}-B_{2}\right)=0, \\
\partial_{t} B_{2}-\sqrt{\frac{\alpha}{\tau}} \partial_{x} B_{2}+\frac{1}{2 \tau}\left(B_{2}-A_{2}\right)=0,
\end{array}\right. \\
\left\{\begin{array}{l}
\left(A_{j}+B_{j}\right)(x, t)=0, \quad j=1,2, \\
\varphi^{\prime}(t)=(\rho H)^{-1}\left(\sqrt{k_{1} / \tau} A_{1}-\sqrt{k_{2} / \tau} A_{2}\right),
\end{array}\right. \\
A_{j}(x, 0)= \\
A_{j 0}(x), \quad \text { in } \varphi(t)<x<x_{0}
\end{gathered}
$$

For (4.8)-(4.12), we have the following global result. 
Theorem 4.2. Assume $A_{j 0}, B_{j 0}, T_{j \#}$ are all $C^{\infty}$ functions and $C^{\infty}$ compatible conditions are satisfied at $(0,0)$ and $\left( \pm x_{0}, 0\right)$. If

(i) $(-1)^{j+1} T_{j \#}>0,(-1)^{j+1} T_{j \#}^{\prime} \geq 0$,

(ii) $A_{j 0}^{\prime}<0, B_{j 0}^{\prime}<0$,

(iii) $|\varphi(0)|<\sqrt{\alpha / \tau}$, then (4.8)-(4.12) has a unique $C^{\infty}$ solution in $[0, \infty)$.

Proof of Theorem 4.2. As in $\S 3$, it is obvious that the key issue for the global existence is the proof of the fact $\left|\varphi^{\prime}\right|<\sqrt{\alpha / \tau}$. As before, we have $\mathscr{A}_{j}=\partial_{x} A_{j}$, $\mathscr{B}_{j}=\partial_{x} B_{j}(j=1,2)$ satisfying the following:

$$
\begin{aligned}
& \left\{\begin{array}{l}
\left(\partial_{t}+\sqrt{\alpha / \tau} \partial_{x}\right) e^{t / 2} \mathscr{A}_{1}=\frac{1}{2 \tau} e^{t / 2} \mathscr{B}_{1}, \\
\left(\partial_{t}-\sqrt{\alpha / \tau} \partial_{x}\right) e^{t / 2} \mathscr{B}_{1}=\frac{1}{2 \tau} e^{t / 2} \mathscr{A}_{1},
\end{array} \quad-x_{0}<x<\varphi(t),\right. \\
& \left\{\begin{array}{l}
\left(\partial_{t}+\sqrt{\alpha / \tau} \partial_{x}\right) e^{t / 2} \mathscr{A}_{2}=\frac{1}{2 \tau} e^{t / 2} \mathscr{B}_{2}, \\
\left(\partial_{t}-\sqrt{\alpha / \tau} \partial_{x}\right) e^{t / 2} \mathscr{B}_{2}=\frac{1}{2 \tau} e^{t / 2} \mathscr{A}_{2},
\end{array} \quad \varphi(t)<x<x_{0},\right. \\
& \mathscr{A}_{1}=\mathscr{B}_{1}-\sqrt{\frac{c_{1} \rho \tau}{\alpha}} T_{1 \#}^{\prime}(t), \quad \text { on } x=-x_{0}, \\
& \mathscr{B}_{2}=\mathscr{A}_{2}-\sqrt{\frac{c_{2} \rho \tau}{\alpha}} T_{2 \#}^{\prime}(t), \quad \text { on } x=x_{0} \text {, } \\
& \left\{\begin{array}{l}
\mathscr{B}_{1}=\left(\frac{\sqrt{\alpha / \tau}-\varphi^{\prime}}{\sqrt{\alpha / \tau}+\varphi^{\prime}}\right) \mathscr{A}_{1}, \\
\mathscr{A}_{2}=\left(\frac{\sqrt{\alpha / \tau}+\varphi^{\prime}}{\sqrt{\alpha / \tau}-\varphi^{\prime}}\right) \mathscr{B}_{2},
\end{array} \quad \text { on } x=\varphi(t) .\right.
\end{aligned}
$$

The same argument as in $\S 3$ shows that under the initial assumption of Theorem $4.2, \mathscr{A}_{j}, \mathscr{B}_{j}$ will remain negative where a $C^{\infty}$ solution exists, or equivalently, where $\left|\varphi^{\prime}\right|<\sqrt{\alpha / \tau}$.

Let $t_{0}$ be the value of $t$, such that

$$
\lim _{t \rightarrow t_{0}}\left|\varphi^{\prime}\right|=\sqrt{\alpha / \tau} .
$$

First, by the relation

$$
\rho H \varphi^{\prime \prime}(t)=c_{1} \rho\left(\left(\varphi^{\prime}\right)^{2}-(\alpha / \tau)\right) \partial_{x} T_{1}-c_{2} \rho\left(\left(\varphi^{\prime}\right)^{2}-(\alpha / \tau)\right) \partial_{x} T_{2}-\rho H \varphi^{\prime}(t),
$$

we see that at $t=t_{0}$, one would have

$$
\sum\left(\left|\partial_{x} A_{j}\right|+\left|\partial_{x} B_{j}\right|\right)=\infty
$$

If not, then $\left|\partial_{x} T_{1}\right|+\left|\partial_{x} T_{3}\right|$ would be bounded near $t=t_{0}$, and $\varphi^{\prime \prime}(t)$ would have opposite sign to $\varphi^{\prime}(t)$ and be of the same magnitude. This is in contradiction to the assumption $\left|\varphi^{\prime}\right| \rightarrow \sqrt{\alpha / \tau}$.

From the condition (4.17), if $\varphi^{\prime} \rightarrow \sqrt{\alpha / \tau}$, then $\mathscr{A}_{1}, \mathscr{B}_{1}$ should be bounded, only $\mathscr{A}_{2}, \mathscr{B}_{2}$ may tend to $-\infty$, i.e., $\partial_{x} T_{1}$ is bounded and $\partial_{x} T_{2} \rightarrow-\infty$. Therefore (4.2) 
implies $\varphi^{\prime \prime}<-\varphi^{\prime}$, which is impossible. Similarly, if $\varphi^{\prime} \rightarrow-\sqrt{\alpha / \tau}, \mathscr{A}_{2}, \mathscr{B}_{2}$ would be bounded, only $\mathscr{A}_{1}, \mathscr{B}_{1}$ may tend to $-\infty$. Hence (4.18) implies $\varphi^{\prime \prime}>-\varphi^{\prime}$, which leads again to a contradiction.

From the above analysis, one sees that it is impossible to have

$$
\lim _{t \rightarrow t_{0}}\left|\varphi^{\prime}(t)\right|=\sqrt{\alpha / \tau}
$$

for any $t_{0}$. This completes the proof of Theorem 4.2.

Remark. One can get the same results for the Cauchy problem when there is no fixed boundary condition (4.14).

Acknowledgment. The author would like to thank Professors V. Alexiades, J. Greenberg, and R. Showalter for sending him the (p)reprints of their papers on the hyperbolic Stefan problem.

\section{REFERENCES}

[1] R. Courant and D. Hilbert, Methods of Mathematical Physics, vol. II, Interscience Publishers, 1962

[2] J. Greenberg, A hyperbolic heat transfer problem with phase changes, IMA J. Appl. Math. 38, 1-21 (1987)

[3] M. Kopàčková-Suchá, On the weakly nonlinear wave equation involving a small parameter at the highest derivative, Czech. Math. J. 19(94), 469-491 (1969)

[4] Ta-tsien Li and Wen-ci Yu, Boundary Value Problems for Quasilinear Hyperbolic Systems, Duke Univ. Math. Series V, 1985

[5] R. E. Showalter and N. J. Walkington, A hyperbalic Stefan problem, Quart. Appl. Math. 45, 769-781 (1987)

[6] A. Solomon, V. Alexiades, D. Wilson and J. Drake, On the formulation of a hyperbolic Stefan problem, Quart. Appl. Math. 43, 295-304 (1985)

[7] A. Solomon, V. Alexiades, D. Wilson and J. Greenberg, A hyperbolic Stefan problem with discontinuous temperature, ORNL-6216, March 1986

[8] Yan Chun Zhao, Boundary value problem for first order quasilinear hyperbolic systems, Chin. Ann. Math. 7A, 630-643 (1986) 